\title{
El canto coral: una mirada interdisciplinar desde la educación musical
}

\author{
Choral Singing: an Interdisciplinary View from Music Education \\ O canto coral: um olhar interdisciplinar desde a educação musical
}

\author{
Santiago Pérez-Aldeguer ${ }^{a}$ \\ aUniversidad de Zaragoza, Facultad de Educación, Departamento de Didáctica de la Expresión Musical, \\ Plástica y Corporal. C/San Juan, Bosco n7, 50009 Zaragoza. Correo electrónico: aldeguer@unizar.es
}

\begin{abstract}
El presente artículo pretende analizar los beneficios que el canto coral aporta a la educación en general y a la educación musical en particular. Bajo este objetivo se examinan numerosos estudios científicos que desde la medicina, la etnomusicología o la psicología, corroboran los aspectos positivos que el canto coral acarrea para la educación. De esta forma se pone a disposición una amplia recopilación bibliográfica, clasificada y seleccionada para dicho fin, con especial atención a las investigaciones de los últimos años. Con la intención de abordar la temática con la mayor claridad posible, se ha divido el trabajo en dos apartados. En primer lugar, se consideran los beneficios que tiene el canto coral para la educación general, a partir de estudios médicos, psicosociales e interdisciplinares. En segundo lugar, se abordan los aspectos positivos de la práctica coral para la educación musical, desde la estética, la psicología y la medicina.
\end{abstract}

RESUMEN

Palabras clave: pedagogía del canto coral, educación general, educación musical, interdisciplinariedad.

\begin{abstract}
This article analyzes the benefits that choral singing brings to education in general and music education in particular. Under this aim are examined numerous scientific studies from medical, ethnomusicology, and psychology, that confirm the positive aspects that choral singing brings to education. Thus, this paper provides a comprehensive bibliography, classified and selected for that purpose, with special attention to investigations of recent years. In order to address the issue as clearly as possible, we have divided the work into two sections. First, the benefits that choral singing brings to general education, from medical, psychosocial and interdisciplinary studies are considered. Second, the positive aspects of choral practice for music education, from aesthetics, psychology and medicine are addressed.
\end{abstract}

Key words: choral pedagogy, general education, music education, interdisciplinary approach.

\section{RESUMO}

Analisam-se os benefícios do canto coral na educação, em geral, e na educação musical, em particular. Sob este objetivo, examinam-se inúmeros estudos científicos que, desde a medicina, a etnomusicologia ou a psicologia, corroboram os aspectos positivos do canto coral para a educação. Desta forma, fornece-se ampla bibliografia, classificada e selecionada para este fim, com especial atenção às investigações dos últimos anos. A fim de abordar a temática com a mior clareza possível, o trabalho divide-se em duas seções. Na primeira, consideram-se os benefícios do canto coral na educação geral, a partir de estudos médicos, psicossociais e interdisciplinares. Depois, abordam os aspectos positivos da prática coral para a educação musical, desde a estética, a psicologia e a medicina.

Palavras chave: pedagogia do canto coral, educação geral, educação musical, interdisciplinaridade. 


\section{INTRODUCCIÓN}

Unos de los principales objetivos de cualquier acto de aprendizaje es su utilidad para el futuro. Partiendo de dicha premisa, en numerosas ocasiones se ha cuestionado el valor de la educación musical en el currículo de educación obligatoria. Pero a pesar de que existen numerosos estudios que reafirman los innumerables valores que la educación musical posee, parece ser que la realidad no se pone de acuerdo con la sustantividad de dichas publicaciones. Por ello, con la intención de manifestar los beneficios que el canto coral aporta a la educación en general y a la educación musical en particular, en este trabajo se presenta una revisión de la literatura sobre los diferentes estudios abordados desde y a través del canto coral. Llevaría muchas páginas recoger todos los estudios en cuanto a canto coral se refiere, por este motivo, se ha pretendido focalizar el esfuerzo hacia el ámbito educativo. Por ejemplo, la revista Eufonía ha realizado varios monográficos en torno al canto coral, por lo que se han revisado todos los artículos y se ha seleccionado los considerados de mayor relevancia, generalmente por aportar una visión novedosa al estado de la cuestión. Del mismo modo, en el Choral Journal, revista americana especializada en estudios corales, hasta el 2011 disponía de un total de 4.015 artículos categorizados en 78 categorías distintas, por diferentes áreas temáticas. A continuación se muestran los estratos más relevantes con el número de artículos publicados de acuerdo a Dorsey (2011):

Gráfica 1. Estudios corales en Choral Journal

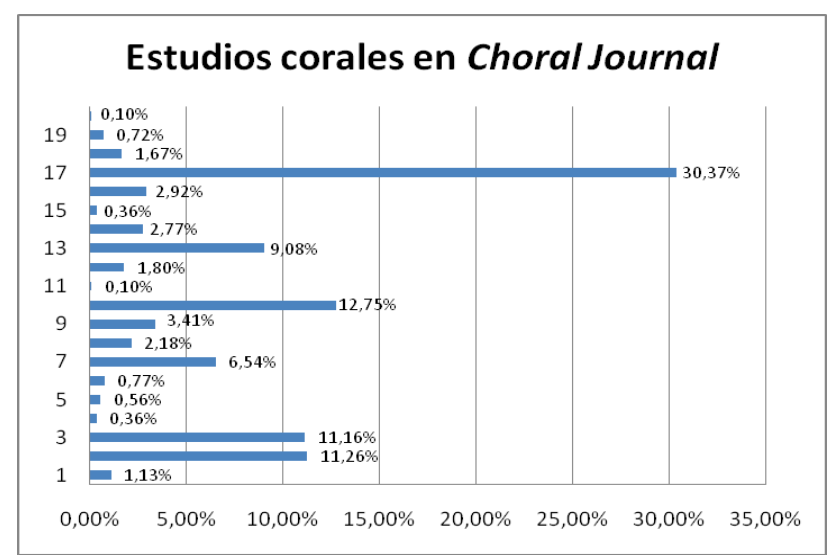

A continuación se muestra la leyenda con el significado de los números que aparecen en la Gráfica 1.

1. Composiciones corales y arreglos.

2. Compositores con su música coral.

3. Dirección coral y técnicas corales.

4. Sobre conciertos.

5. Discografías.

6. Formas musicales de la música coral. 
7. Historia y bibliografías de corales y directores de coro.

8. Historia y análisis de la música coral.

9. Entrevistas.

10. Literatura para varios tipos de coro.

11. Teatro musical.

12. Prácticas y estilos de la interpretación coral.

13. Novedades y actividades corales: 354

14. Filosofía y estética.

15. Grabaciones y técnicas de amplificación y equipamiento.

16. Artículos bibliográficos.

17. Recensiones a libros y a grabaciones.

18. Actividades corales.

19. Textos.

20. Acompañamiento.

Como se observa, no existe categoría alguna que haga referencia a la educación coral y a su influencia en la educación general o musical en cuestión.

\section{BENEFICIOS DEL CANTO CORAL EN LA EDUCACIÓN}

Gackle y Fung (2009) realizaron un estudio donde se documenta y evalúa el proceso llevado a cabo por 35 cantantes de un coro de USA, con edades comprendidas entre los 14 y 18 años, para aprender piezas corales chinas durante un periodo de cuatro meses. La metodología utilizada fue la observación, las entrevistas y la evaluación de la interpretación musical. Los resultados obtenidos se clasificaron en ocho temas principales, entre los que se encuentra: el disfrute de la estética, la comprensión de las letras, la prolongación de su contexto cultural y la facilitación de la cultura. Las conclusiones manifestaron que las estrategias utilizadas para el aprendizaje de dicho repertorio habían sido eficaces, las cuales repercutieron positivamente sobre cuatro áreas de los estudiantes: la musical, la pedagógica, la cultural y de actitud, y la personal.

$\mathrm{Al}$ analizar el método empleado por los profesores y estudiantes para el aprendizaje de las piezas de música coral china, se percibe el gran desarrollo que la educación musical ha sufrido en los últimos años. Por ello, independientemente del método utilizado es importante que el docente transforme el método en una metodología propia, innovadora y flexible.

Tal y como describen Gackle y Fung cuando se trata la adquisición de conocimientos musicales, a nadie extraña que al cantar en un coro estos aumenten. Ahora bien, al hacer referencia a la actitud, surge una pregunta: $¿$ Se puede modificar la conducta de una persona que canta en un coro? Naturalmente, la acción de hacer música posee un conocimiento, un valor intrínseco, que por consiguiente predispone a la persona en cuestión hacia una actitud determinada y como resultado final, esta desemboca en una actitud concreta. Son abundantes los trabajos pedagógicos que destacan la importancia de la transmisión de valores en la educación (Marín, 1976; Touriñán, 2007; Quilaqueo y Rapimán, 2006). Por ello, se han abordado numerosos estudios que demuestran que el hecho de cantar en un coro desarrolla el conocimiento musical, así como otra serie de capacidades que 
benefician en otros aspectos a las personas que lo cultivan. De acuerdo con Touriñán y Longueira (2010: 176): "La formación integral que debe facilitar la educación general queda sesgada e incompleta si descuida las posibilidades de la experiencia artísticomusical como ámbito general de la educación”. Así mismo, Ferrer (2009) evidencia dicha afirmación desde la educación en valores que conlleva el canto coral, en una experiencia en L'Escola de Música de Palafrugell.

Desde el ámbito universitario coral, el trabajo de Sichivitsa (2003) realiza una evaluación sobre la influencia de los padres y el soporte que estos ofrecen a sus hijos. Se trata aspectos del autoconcepto en las habilidades musicales, el valor de la música y la integración académica de los estudiantes en el coro universitario fuera y dentro de la Universidad. En la encuesta participaron 154 estudiantes del coro de una Universidad Pública del sur de EEUU. La investigación se enmarca en un estudio cuasi-experimental donde el $50 \%$ de los participantes tenían como intención la integración social en el coro. Dicho estudio evidencia uno de los valores fundamentales que posee el canto coral: su capacidad inclusiva. Así mismo, la educación inclusiva ha sido ampliamente contextualizada desde los años noventa en la Conferencia Mundial sobre Educación para Todos celebrada en Tailandia. Pero en una formación coral, las personas que la integran deben aprender a escuchar a sus compañeros, aprender a esperar, comenzar en el momento oportuno, con una dinámica, una frecuencia (tono), etc. El hecho de cantar en una agrupación coral desarrolla un modelo educativo inclusivo de forma inherente, como afirma el maestro Abreu (Premio Príncipe de Asturias de las Artes, 2008): "la música es un instrumento irremplazable para unir a las personas".

El canto coral no sólo ha servido como medio para desarrollar o mejorar aspectos de la educación, la medicina o la psicología, sino que también se ha utilizado como herramienta de expresión política (Deniz Silva, 2001). De hecho, pueden encontrarse titulares de periódicos que ratifican esta afirmación, como por ejemplo: "Urkullu apuesta por convocar a la sociedad vasca para hacer un canto coral" (Noticia del 24/10/2011 en el Periódico digital La Vanguardia, consultado en http://goo.gl/KAjfS el 22/12/2011). Mediante la expresión "un canto coral", el presidente del PNV se refiere a la expresión de manera conjunta. En este mismo sentido, y desde la psicología, Hurtado Llopis $(2011 ; 2006)$ presenta diferentes trabajos de reflexión sobre el desarrollo personal y social que el acto de cantar en coro produce para la educación. El autor aborda ampliamente el desarrollo de la asertividad en la Coral Allegro ONCE de Valencia (España), la cual dirige desde su creación en 1982. Fundada desde un colegio de educación especial, se trata de un perfecto ejemplo de la capacidad inclusiva y de los beneficios que esta revoca de forma directa en la educación.

\subsection{BENEFICIO PARA LA EDUCACIÓN VISTO DESDE LA MEDICINA}

Los autores Bygren, Konlaan y Johansson (1996) analizaron la influencia que posee cantar en un coro, hacer música, la lectura y la asistencia a actividades culturales como factor determinante para la supervivencia. Para ello, diseñaron una muestra aleatoria de 15.198 sujetos entre 16 y 74 años. Del total de la muestra el 85\% (12.982) fueron entrevistados por personal no médico entre 1982 y 1983, y realizaron un plan de seguimiento hasta el 31 de diciembre de 1991. Se controlaron ocho variables: edad, sexo, nivel educativo, ingresos, enfermedad, vida social, el tabaco y el ejercicio físico de 6.301 hombres y 6.374 mujeres; se les aplicó un seguimiento donde 533 hombres y 314 mujeres 
fallecieron durante este periodo. Finalmente se encontraron influencias en la mortalidad cuando las ocho variables de control fueron observadas en personas que rara vez asistieron a eventos, en comparación a las personas que asistieron con más frecuencia, siendo el riesgo de 1,57 (confianza del 95\%, 1,18 al 2,09). Con todo ello, se llegó a la conclusión de que la asistencia a eventos culturales tiene una influencia positiva en la supervivencia.

Del mismo modo, se observa en el artículo de Heald (1996), cómo la medicina es capaz de relacionarse con el mundo musical de manera directa. El autor desde la abogacía trata los derechos de autor cuestionando las leyes de propiedad intelectual desde la perspectiva de los arreglistas y los directores de coro.

Desde la musicoterapia y la educación especial, Eyre (2011) nos brinda un artículo titulado: Coral terapéutica para las personas con enfermedad mental crónica: un estudio descriptivo de las experiencias de los participantes. En él se evalúa la relación entre la participación en el coro y la calidad de vida que esto les reporta a los pacientes. La muestra se compuso por 16 personas con enfermedad mental grave y el instrumento utilizado fue un auto informe con un metodología cualitativa para los pacientes, diseñado por los investigadores. Los resultados revelan que los miembros del coro percibían su autoestima, la expresión de emociones, las alteraciones del humor, el enfrentarse al stress y el tener una rutina de una forma positiva. Se puede llegar a pensar que quizás el hecho de cantar en un coro, pueda tener aspectos positivos sólo desde la musicoterapia -teniendo en cuenta las múltiples facetas que la misma posee-, pero los estudios llevados a cabo por Carminatti y Krug (2010) demuestran lo contrario. Se ha hablado mucho de los aportes positivos que la práctica musical en conjunto tiene sobre las competencias sociales de quien la llevan a término, así como del aspecto democrático (en el caso de que la agrupación no disponga de director); los miembros deberán tomar decisiones sobre la dinámica, los comienzos y finales de las piezas, y un largo etcétera. Las autoras quisieron averiguar la existencia de diferencias o no de las habilidades sociales entre adolescentes que cantan frente a otros que no lo hacían. Para ello se hizo uso de la una metodología cuantitativa, con entrevistas semi-estructuradas y la exploratoria comparativa. La herramienta utilizada había sido el IHS - DES PRETTE (Inventario de Habilidades Sociales) y un cuestionario sociobiodemográfico en 100 participantes con edad entre 14 y 17 años. Los resultados manifiestan que la práctica del canto coral repercute en varios momentos y contextos de sus vidas, generando sentimientos y oportunidades, además de promover el desempeño de diferentes habilidades, como la expresión de afecto positivo de los coristas frente a las habilidades de conversación y desenvoltura, que resultaron mayores en los no coristas. Es aquí cuando se nos plantea la pregunta: qué beneficios aporta el canto coral a la Psicología Social Comunitaria, la cual persigue el desarrollo de instrumentos de análisis e intervención que ayuden a solucionar problemas que afectan a la sociedad actual.

La música ha tenido un cierto status social en diferentes épocas históricas, se ha utilizado para diferentes fines entre ellos el terapéutico. Los egipcios, los griegos, o los escritos hebreos así lo manifiestan, así mismo tuvo mucha influencia en el Medievo y el Renacimiento (Hatem, Lira y Mattos, 2006). Pasaron diferentes etapas de altibajos, hasta que finalmente se consideró que la música era lo sumamente importante como para estar dentro del currículo de educación obligatoria. La formación coral en la educación obligatoria resulta sugerente e imprescindible por diversas razones; quizás una que prácticamente no conlleva demasiada controversia en sí, es la posibilidad de pensar 
que todos disponemos de una voz para utilizarla. No sólo la voz hablada sino también la voz cantada, pues con ella se puede abarcar cualquier estilo/género musical, lo cual tiene un componente fuertemente inclusivo y democrático.

Pero también ha existido un nexo de unión entre el canto coral y el body music (música con el cuerpo). Terry (1989) la define como la música/danza creada con el cuerpo a través de palmadas, pasos y vocalización, o los sonidos de percusión con la voz (beatbox). Diversos artículos publicados por Javier Naranjo en la revista Música y Educación tratan dicha temática. Por ello, De Souza y Dos Santos (2009) aplican estas técnicas en el ámbito de la musicoterapia, dentro de un proyecto titulado: The Body in Music, The Music in Body: a community integration project, el cual utiliza el acto de cantar en un coro y las actividades terapéuticas con música (body music) en personas con Parkinson. Entre los objetivos del proyecto se encuentran aspectos tales como: la mejora de la autoestima, la disminución de los niveles de depresión, trabajar la coordinación motora, desarrollar la percepción musical, la sociabilización, fomentar la relajación y mejorar la expresión facial. Aunque el proyecto todavía no ha finalizado, se ha demostrado que los temblores disminuyen cuando el grupo está envuelto en las actividades de música con el cuerpo.

\subsection{BENEFICIO PARA LA EDUCACIÓN DESDE UN PUNTO DE VISTA INTERDISCIPLINAR Y PSICOSOCIAL}

Los estudios en torno al mundo coral abordan múltiples disciplinas, algunos de ellos desde distintos ejes transversales inseparables, aportan a la educación general valores imprescindibles para los educandos. Por ejemplo, en estudios realizados desde la musicología, concretamente se trata la música del coro de la iglesia Preštice en Bohemia desde el siglo XVIII hasta la actualidad (Fiala, 1996). Otros autores como Bohlin (1993); Jurado Luque (2010) o Sancho García (2007) parten desde el pilar del canto coral hacia la música, desde donde se cultiva la historia y la geografía, sin perder de vista el gusto por la estética y la cultura popular. Este tipo de estudios, nos aportan una visión interdisciplinar de la materia, ya que la educación no puede ser vista como compartimentos estancos, sino que existen unas dentro de otras. La interdisciplinariedad hoy en día se ha convertido en una necesidad, la sociedad actual se ha venido desarrollando hacia un modelo globalizado, "obligándonos" en ocasiones a trabajar juntos para conseguir metas comunes. Este hecho lo observamos en los grupos de investigación actuales; es necesario numerosas disciplinas aparentemente poco afines entre ellas para llevar a cabo diversas tareas dentro del mismo proyecto. La interdisciplinariedad ayuda a resolver numerosos problemas sociales de los que inevitablemente, debe contemplar aspectos psicológicos, culturales, sociales y tecnológicos, entre otros. Este modo de trabajo se está convirtiendo en una necesidad del mercado laboral actual, donde el éxito está muy relacionado con la capacidad de trabajo en grupo. Siendo desde la interdisciplinariedad, donde el canto coral aporta elementos significativos a la educación general.

Por otro lado, Walter (1992) realiza un estudio sobre los coros angélicos, creencias individuales y motivos personales de los cantantes de coro, para una interpretación sociológica de la non secularization de la música coral. Con el término non secularization se refiere a la transformación de una sociedad que parte de valores religiosos hacia los no religiosos; la religión pierde su autoridad en la vida social. En el presente estudio, se observa como la religión se ha servido de la música para diferentes fines y como ella 
nos podría servir para el conocimiento de la primera, a través de su repertorio vocal. La literatura musical religiosa ha utilizado mayoritariamente la voz para la transmisión de sus fines. Desde la etnomusicología y conectado con el mundo coral, la investigación abordada por Seroff (1990) realiza un primer acercamiento a los coros zulú, un grupo étnico de la provincia de KwaZulu-Natal en Sudáfrica. Desde dicha vertiente se aprecia el importante papel que tiene la elección del repertorio coral para un director de coro, y cómo a través de una canción se tratan diversas materias educativas.

Desde la interdisciplinariedad, el repertorio coral en beneficio de una educación intercultural ha sido tratado por Ortiz Molina (2011a), quien manifiesta que las publicaciones en revistas de educación musical han centrado su interés en la enseñanza de la música desde una sociedad multicultural. En Andalucía, nos dice la autora, se viene planteando desde hace años un modo de abordar la política de inmigración que arranca de una visión integral del fenómeno, cuyo fin último es la plena incorporación de la persona inmigrante. Uno de los desafíos a los que un docente de música se enfrenta a la hora de abordar la interculturalidad, es la utilización de cualquier estilo/género musical para el desarrollo de conceptos, valores, actitudes y conductas. La educación intercultural nos lleva a romper el cliché del alumno modelo, en pro de la diversidad y el reconocimiento de la individualidad. En esta misma línea, se encuentran otros proyectos como el llevado a cabo en Noruega con 10 maestros y 20 estudiantes; el objetivo había sido conocer los pensamientos de los estudiantes sobre la autoeficacia y la empatía en torno con la interculturalidad. El resultado obtenido fue que, desde el aprendizaje acción, se ayuda a los profesores y estudiantes a obtener mejor comprensión de las diferentes costumbres culturales (Ortiz Molina, 2011b). La interculturalidad ha sido planteada con canciones de diferentes lugares, desde una perspectiva social de la música que contribuye a la sociedad y la cultura (Regelski y Gates, 2009).

Desde una mirada musicológica y etnomusicológica el artículo del autor alemán Heimbucher (2009), habla sobre la música coral espiritual de Louis Spohr. El enfoque del autor es un ejemplo para un tratamiento futuro de la interculturalidad desde la diversidad. Así también, el ensayo escrito por Dueck (2008) es un intento de introducir a los lectores al canto coral menonita, mediante grabaciones del West Coast Mennonite Chamber Choir, resultando sugerente en su planteamiento, pero quien aporta a la temática un tratamiento divergente. Así mismo, existen numerosas investigaciones que se incluyen en el apartado dos y tres respectivamente. Es el caso de Elorriaga (2011) y Freer (2010), quienes estudian la construcción de la identidad de género en los adolescentes, mediante un estudio de caso y utilizando metodologías cuantitativas y cualitativas. Los autores poseen diversas publicaciones al respecto sobre la misma temática, con un tratamiento algo distinto, bajo el objetivo de manifestar la relación existente entre la identidad vocal y el género en estudiantes de Educación Secundaria. Las conclusiones extraídas manifiestan las implicaciones positivas que el canto coral posee en la construcción de la identidad de género en los varones adolescentes.

Desde una mirada desemejante, MacLellan (2011) realiza un estudio explorando las diferentes personalidades existentes entre un grupo de 355 estudiantes de educación secundaria que formaban parte de la banda, la orquesta de cuerdas y el coro de su instituto. Como instrumento de medida utiliza el cuestionario: Myers-Briggs Type Indicator. Las conclusiones ponen de relieve que existen diferencias entre los miembros de las tres formaciones, pero la significación viene dada por los estudiantes de coro que eran más 
extrovertidos en comparación con los de la orquesta. Por otro lado, desde la interdisciplinariedad y la transversalidad, Carlow (2006) aporta un estudio de caso llevado a cabo con alumnos de secundaria que cursaban la asignatura de coro e inglés, con el objetivo de examinar las percepciones de los cinco alumnos inmigrantes que cantaban en el coro y la influencia de ello para el aprendizaje del inglés. Finalmente, el trabajo de Dwiggins (1984) trabaja el canto coral en su relación con la educación física, la psicomotricidad y el movimiento, ofreciendo sugerencias de movimientos coreográficos para coros, considerando habilidades y tamaño del grupo.

\section{BENEFICIOS DEL CANTO CORAL PARA LA EDUCACIÓN MUSICAL}

Las aportaciones que el canto coral reporta a la educación han sido también abordadas a través de la organización de Jornadas, Congresos, Foros...destacando en el 2011 el: "I Congreso de Música Coral en Centros Educativos" y las I Jornadas Corales, ambas celebradas en Valencia (España). Esta última está recogida en el libro Contextos corales. Así mismo, Sotelo (2002; 2009), reúne en dos artículos los encuentros corales de enseñanza secundaria celebrados en Cataluña, con el propósito de que la música se convierta en una fuerza motivadora en la vida de los alumnos. Del mismo modo que con la intención de mejorar la formación vocal de los futuros maestros de educación musical, Elsgtröm (2009) propone algunas recetas para el cambio. Ello hace que se investigue y se conozca que el canto coral tiene repercusiones positivas en la educación musical y general del ser humano. El ISME (International Society for Music Education), el Encuentro Regional Latinoamericano ISME, el NAFME en USA (The National Association for Music Education), el EAS en Europa (European Association for Music in Schools) son entre otros organismos, los encargados de establecer espacios de debate y difundir investigaciones al respecto.

Existen numerosos prolegómenos en torno al canto coral, que facilitan la inclusión en la educación para y a través de la música. Por ejemplo, el libro titulado Teoría y práctica del canto coral de Miguel-Ángel Jaraba, propone un acercamiento a cantores y directores de coro, al conocimiento de una cultura coral básica y al mundo coral apta para eruditos y profanos. Brünner (2009) u Ortega Castejón (2005) presentan sencillos libros, artículos y manuales para distintos niveles como introducción al mundo coral. La recopilación de repertorio popular para voz, los cancioneros, ha sido materializado en trabajos como los de Gómez Gallego y Rodilla León (2009); y ampliamente desarrollados en diferentes publicaciones en revistas, libros y diferentes estudios musicológicos. Desde el punto de vista de la musicología se han realizado multitud de estudios referentes que toman como punto de partida el canto coral, siendo el caso de Quijada Sagredo (1985), desde donde se percibe el tratamiento interdisciplinar que se le da al objeto de estudio en el artículo El canto coral en la tragedia tardía de Eurípides. Desde esta misma perspectiva el estudio de Morales Villar (2007), Ramón Guitart-Besangèe y El arte de desarrollar la voz y sus timbres (ca. 1915): La escuela de canto de Manuel García en Buenos Aires. Dichas publicaciones son complementarias al estudio de Carbonell I Guberna (2003), donde presenta una reflexión sobre el recorrido histórico coral en España, resaltando el aspecto de la sociabilidad coral, ampliamente abordada por diversos autores mencionados en el apartado anterior. 
Por otro lado, existen numerosos artículos en alemán sobre la materia, destacando algunos publicados en la revista Musik und Kirche, como por ejemplo Música antigua práctica interpretativa para coros amateurs de Brodel (2003); Música góspel para corosuna lista anotada de los materiales básicos de Teichmann (2003); Los coros y los lugares donde se canta - Un estudio de la música coral contemporánea británica de Gant (2001) y Experimentar la música en la carne. Algunas ideas pioneras de una forma diferente de trabajar con coros de Kotter (2000). Por otra parte resulta sugerente la propuesta del estudio llevado a cabo por Schweizer (1989), quien examina la educación musical elemental y la improvisación en el coro infantil, bajo un planteamiento poco tratado hasta la fecha. Así mismo, Reames (2001) realiza encuestas a 263 directores de coros de secundaria, poniendo de manifiesto que los directores, que son quienes seleccionan el repertorio para coros de iniciación y de alto nivel, dan mucho valor a los conciertos, así como a las librerías corales personalizadas que se crean y por ende a las grabaciones corales.

En el ámbito de la educación secundaria, Daniels (1986) presenta un estudio para encontrar la relación entre la capacidad de repentización (lectura de una partitura musical a primera vista) de los 20 coros mixtos de 20 institutos de secundaria, en relación a cuatro variables dependientes: escuela, currículo de música, profesor de coro, estudiantes. Se corrobora que la composición multicultural del aula, el disponer de un piano en casa y una escuela rural, mejora la lectura a prima vista de los educandos. Abordando la temática coral desde el lenguaje musical el artículo de Major (1982), trata el efecto de la actividad de la subdivisión de las destrezas de interpretación rítmica de 3 coros mixtos de institutos de educación secundaria. La metodología fue cuantitativa pre-post test (grupo experimental y control), encontrando diferencias significativas $(\mathrm{p} \leq 0.01)$, a favor de la subdivisión para una mejor interpretación de ritmos con precisión.

\subsection{QUÉ MÚSICAS UTILIZAMOS PARA LA EDUCACIÓN A TRAVÉS DE LA MÚSICA}

Touriñán y Longueira (2010), en su artículo titulado La música como ámbito de educación. Educación por la música y para la música, clarifican de una forma precisa la diferencia existente entre la educación por la música y la educación para la música. La primera es aquella educación musical que adquiere un significado global para el desarrollo de la persona a todos los niveles, constituyéndose como una verdadera educación holística. La segunda, educación para la música, está pensada más para las personas que se dedicarán en un futuro a la música de forma profesional. Partiendo de esta perspectiva, Bass (1982) analiza el papel de los coros de música popular en los centros educativos de Primaria y Secundaria, así como en las Universidades. El autor afirma que hacer coros pop es un excelente método para la enseñanza de habilidades para el canto y la teoría musical. Y es aquí donde nos surge la pregunta: ¿De qué músicas nos servimos en el aula para educar por la música? ¿Qué músicas incluimos en el aula? La palabra inclusión de "otras músicas" en los centros educativos está considerada en la actualidad como un paradigma, el cual es ampliamente tratado en diferentes congresos internacionales como los organizados por el ISME bianualmente.

En este mismo sentido, Stanley (1970: 8) afirma que "Tradicionalmente los profesores han hecho caso omiso de los intereses musicales actuales de los alumnos". Por ello, en los años 70 en Estados Unidos se llevó a cabo un estudio en el ámbito de la educación 
obligatoria en torno al desarrollo de la comprensión musical en las técnicas compositivas de las músicas "modernas" del S.XX. La investigación se desarrolló a lo largo de dos años en cinco escuelas de secundaria de Nueva York; las músicas utilizadas fueron el rock, pop, soul, música electrónica y jazz. El objetivo fue buscar la relación que existía entre esas músicas y otros estilos como la música impresionista o expresionista, entre otros. El resultado que se obtuvo fue una visión general y unas guías que se elaboraron para que los maestros de música pudieran incluir diferentes repertorios de música, sin que ninguna resultara excluyente. Cierto es que como se ha discutido en diferentes foros sobre educación musical, el discente está expuesto a un gran número de horas a estilos como el pop, rock, etc. Por ello en pro de la equidad de estilos, se debe poder disponer de investigaciones sólidas, creativas y motivacionales para incluir en el aula diferentes estilos/géneros musicales, dentro de un balance y secuenciación didáctica lógica.

\subsection{APORTACIONES DESDE LA PSICOLOGÍA}

Phillips (1982) propone una investigación llevada a cabo mediante entrevistas a diferentes directores de coros góspel, el objetivo es describir algunas percepciones que éstos tienen hacia dicho estilo/género musical. Desde la educación, Gronostay (1980) ofrece un artículo en alemán sobre tareas y objetivos que tiene un director de coro. Por otro lado, Wills (2011) en su estudio se centra en tres aspectos sobre la espiritualidad y el bienestar en relación con la experiencia de hacer música: la transcendencia, la conexión y el flujo. Para ello, centra su trabajo en la creación musical, concretamente, en la actividad de cantar en un coro. Se trata de una metodología por observación con niños de 5 a 11 años de edad. Los resultados expusieron el cambio de comportamiento y mejora de la autoestima de algunos niños de la escuela, desde que forman parte del coro, mejorando otras áreas de la vida escolar.

Así mismo, las investigaciones de Hampshire y Matthijsse (2010) buscan corroborar si los proyectos artísticos mejoran el bienestar de los jóvenes. Para ello, focalizan el objetivo en el bienestar social y emocional, en relación con el capital social, siendo ésta la causa más importante por lo que se considera la participación en las artes, buena para la salud. Se parte de la premisa de que los proyectos artísticos en comunidades tienen un impacto positivo en la inclusión, la salud y el bienestar. La metodología utilizada ha sido por observación participante, las entrevistas y las encuestas, donde se examina la experiencia de los niños en tres coros. Los resultados manifiestan que para los niños de familias relativamente privilegiadas, la experiencia es muy positiva, hacían nuevos amigos y la experiencia les generaba confianza. Pero los autores sostienen que mientras que los proyectos artísticos pueden tener un impacto positivo en el bienestar social y emocional, no podemos suponer que los cambios serán sencillos. En el artículo analizan las posturas de diversos teóricos críticos, quienes argumentan que el capital social está altamente relacionado con el capital económico y cultural.

Por otro lado se encuentran autores como Bowers (1998), quien persigue el objetivo de examinar las aptitudes de los estudiantes universitarios y personas mayores hacia los demás, mediante la incorporación de aspectos que funcionaron en el programa de música para la tercera edad "Adopt-A-Coro". Con una metodología cuasi-experimental y la utilización del Grupo de Evaluación de Edad y el Inventario de Descripción (DE), se proporciona información sobre las actitudes clasificadas en cuatro dimensiones: La 
bondad, la positividad, la vitalidad y madurez. Los participantes fueron 15 personas del coro de mayores y 12 estudiantes de educación musical. Los resultados de la encuesta EDAD pre/post se compararon mediante la prueba de Wilcoxon de pares coincidentes, con los medios para aumentar significativamente los cuatro dominios $(\mathrm{p}<.02)$. Los resultados manifiestan que las ganancias fueron mayores para las personas en la tercera edad y menores para los estudiantes universitarios, pero todos positivos.

Por otro lado, el estudio de Gumm (2004) analiza el efecto que tiene para el aprendizaje y la motivación de los estudiantes de coro, el estilo de aprendizaje que se utiliza. El objetivo ha sido comprobar si el estilo de aprendizaje influencia en el coro y si las herramientas para medir el constructo fueron fiables. Con un total de 273 estudiantes de coro de educación secundaria y utilizando el aprendizaje de Kolb (basado en experiencias), inventario de estilos de aprendizaje y otros, se tomaron en consideración la motivación, el estilo de aprendizaje y el estilo de enseñanza de la música. Resaltándose que la motivación por la música predijo una variación moderada en cada dimensión de la música por cada uno de los estilos de aprendizaje utilizados.

Por otro lado, Lobos (2008) también trata la temática coral de forma más indirecta, pero desde un punto de vista más musical que los anteriores. Con una grabación de la obra del compositor polaco Witold Lutoslawski 20 villancicos polacos para soprano, coro femenino y orquesta, el autor utiliza la obra como un ejemplo muy bueno de las interacciones pedagógicas en la escuela de música polaca. Concluye expresando que este ha sido un buen material para el trabajo entre compañeros y un buen ejemplo de las tradiciones conservadas por las naciones. El estudio tuvo lugar en la Karol Szymanowski State Secondary Music School in Katowice. Por otra parte, el estudio llevado a cabo por Cámara (2003) con el objetivo de conocer el grado de aceptación que los alumnos de $3^{\circ}$ a $6^{\circ}$ de Primaria de siete colegios de Bilbao $(\mathrm{N}=367)$ tienen a la hora de realizar las actuaciones de canto colectivo. Casi el $100 \%$ de los encuestados tuvieron una actitud positiva sobre el hecho de participar en conciertos didácticos y preparar las canciones con el grupo. Lo que se evidencia con este trabajo una vez más es la capacidad social y la capacidad de cohesión del canto coral. Como se ha demostrado, el canto tiene la posibilidad de aumentar la autoestima y como no podía ser de otro modo, interviene en la construcción de una identidad propia dentro del grupo; por ello, es una herramienta apta para la educación por y para la música.

\subsection{APORTACIONES DESDE LA MEDICINA}

Clift y Hancox (2001) nombran los beneficios del canto tras un estudio de caso llevado a cabo con una coral universitaria, donde los 84 miembros completaron un cuestionario. En él se les preguntaba en torno al beneficio personal en su participación en el coro y como dicha participación beneficiaba a su salud. El $87 \%$ declaró que habían beneficios sociales, el $75 \%$ emocionales y el $58 \%$ contestó que había beneficio de alguna manera, contestando el $49 \%$ que existía un beneficio físico y espiritual. Así mismo, se realizó un informe de contenidos para ver las formas en los que los miembros del coro sintieron que se habían beneficiado. Los temas expresados comúnmente fueron: conocer gente nueva, tener un mayor control sobre la respiración, tener un mejor estado anímico y reducir el estrés. El segundo cuestionario fue realizado por 91 miembros de otro coro; este constaba de 32 cuestiones sobre el canto que ya reflejaba el anterior estudio. Más 
del $40 \%$ estuvo de acuerdo en que el canto ayuda a tener un estado anímico más positivo, por ejemplo, con el discurso de cantar me hace sentir más feliz, etc. Se realizó un análisis de componentes principales, seguido por la rotación Oblimin, identificando seis dimensiones de beneficio asociado con el canto. Estos fueron etiquetados como: beneficios para el bienestar y la relajación, para la respiración y la postura, las prestaciones sociales, espirituales, emocionales y para el corazón y el sistema inmunológico. Se aplicó el coeficiente del alfa de Cronbach siendo satisfactorios para todos los componentes, excepto para los beneficios sobre las prestaciones sociales, debido principalmente al escaso número de elementos de carga en este componente. Resulta curioso que las mujeres fueron significativamente más propensas a experimentar los beneficios para el bienestar y la relajación, y las personas que profesan creencias religiosas eran más propensas a experimentar los beneficios espirituales.

Por otro lado, Griffiths (2008) nos presenta un tema no menos relevante: la sordera de tono. Durante mucho tiempo las personas a las que se les diagnosticaba sordera de tono, no eran aptas para la música y mucho menos para cantar en un coro. La sordera de tono se considera como un trastorno en la producción; estudios recientes lo han clasificado como trastorno cognitivo: agnosia auditiva. Este artículo analiza cómo el fenotipo (características observables) construye el nivel de déficit causal de la percepción del tono. Este trastorno resulta interesante porque ofrece la oportunidad de estudiar cómo un complejo fenotipo puede ser caracterizado como un trastorno de percepción cortical potencialmente explicado por un solo gen o molécula. A pesar de que la sordera de tono no es muy perjudicial, la anomalía subyacente puede llegar a ser un trastorno de la conectividad cortical que proporciona un modelo para los trastornos como la esquizofrenia.

\section{ALGUNAS CONSIDERACIONES FINALES}

En el presente trabajo se ha pretendido realizar una recopilación sobre diferentes estudios que revelan el beneficio que el canto coral aporta a la educación en general y a la educación musical en particular. Se observa que los beneficios relacionados con la educación general, los cuales se abordan en el epígrafe dos, son numerosos. La educación musical pretende proporcionar a los estudiantes una salida a las experiencias de aprendizaje después de su educación formal y promover el uso de conocimientos previos para el enriquecimiento a lo largo de toda su vida. Como se ha podido comprobar, existen numerosas investigaciones que a pesar de no tratar la temática coral como eje principal, han utilizado la misma para investigaciones desde otras áreas de conocimiento, tales como: la medicina, el derecho, la sociología o la psicología. Así mismo, resulta alentador corroborar cómo las investigaciones más numerosas se realizan desde estas disciplinas. De este modo, nos acercamos una vez más a una enseñanza transversal e interdisciplinar. La interdisciplinariedad en el ámbito de la educación musical, ha sido ampliamente abordada entre otros autores por Ruthmann et al. (2010), Lobos (2009), Grant et al., (2008) y Pérez Aldeguer y Leganés (2012), quienes desarrollan diversas propuestas prácticas, al respecto.

Desde la musicología se puede examinar las tensiones y resoluciones en las melodías y armonías, así como la mezcla viva de la estimulación auditiva. Como sociólogos, se puede tratar de explicar el papel de la música en las sociedades y culturas; y como psicólogos, el efecto que ésta tiene sobre nosotros (Durrant, 2009). La música es un conocimiento 
que está impregnado de numerosos saberes inherente, es decir, no podemos verlos de forma aislada. De este modo, la música nos ofrece numerosas oportunidades para una educación holística en el más amplio sentido de la palabra. Los aspectos sociales encontrados científicamente en la práctica de la música coral la han dotado de una notable valía para la educación general y como no podía ser de otra forma, para la educación musical. En la pirámide de Maslow, la necesidad de afiliación y afecto se encuentran en su tercer estadio. Por ello, las energías creadas en el seno de una interpretación musical, independientemente de que provengan de un contexto, son como señalan Touriñán y Longueira (2010) de ámbito general (por la música) o profesional (para la música). Estas producen una serie de transformaciones en el seno de sus percepciones, pudiendo encontrarse mediante un sistema de comunicación asemántica como es la música, la aceptación o el sentido de pertenencia. La música se comunica ante nosotros en código abierto, pero cuando cantamos en grupo se desarrollan capacidades y cualidades que no son posibles desarrollar con otras actividades. Dos personas hablando al mismo tiempo pueden resultar molestas, sin embargo, dos, tres y cientos de personas cantando al mismo tiempo resulta hermoso.

Es por ello que los proyectos interdisciplinares en el ámbito musical, también se han desarrollado en la Educación Superior. Un ejemplo de ello lo encontramos en un proyecto donde la música y la informática, mediante una metodología de trabajo por servicio, se estrechan la mano (Omishakin et al., 2009). Del mismo modo, se han desarrollado diversos proyectos en nuestro país que, todavía sin llegar a ser estudios científicos por no disponer de las herramientas propias de la investigación, han demostrado ser fuente de inspiración para futuras iniciativas similares. Entre otros se destaca el Proyecto LOVA, una iniciativa que intenta realizar un proyecto globalizado, en cooperación con los distintos maestros de primaria. Está centrado en la creación de una ópera, donde los niños/ as elaboran toda la obra de principio a fin (vestuarios, textos, música, etc.), adquiriendo con ello las competencias básicas que se le demandan a sus docentes en el ámbito de la educación obligatoria. La competencia cultural y artística solicita una presencia mayor en la escuela y ésta ha sido ampliamente elaborada por Giráldez (2007), quien posee diversas publicaciones al respecto.

Como se mencionó anteriormente, a pesar de que las investigaciones sobre educación musical han medido los beneficios que tiene la continuidad en la práctica musical a lo largo de la vida, en lo que a músicos aficionados se refiere, se observa una contradicción en la presencia de esta en los currículos. Quizás por ello se puede decir que "La inclusión de las artes, en general, y de la música, en particular, en los sistemas educativos tiene relación con el modelo de ser humano y el modelo de sociedad que queremos" (Touriñán y Longueira, 2010: 53). Pero no por ello se invalida o excluye los numerosos beneficios que desde diferentes perspectivas han iluminado la práctica coral para la educación general y para la educación musical en particular.

\section{REFERENCIAS BIBLIOGRÁFICAS}

Bass, LP. (1982). In the swing of things. Music Educators Journal, vol.68, n.6, 49-62.

Bohlin, F. (1993). Investigaciones sobre el canto coral. Revista de musicología, vol.16, n.2, 1062-1064.

Bowers, J. (1998). Effects of an intergenerational choir for community-based seniors and college students on age-related attitudes. Journal of Music Therapy, vol.35, n.1, 2-18. 
Bygren, L., Konlaan, B., y Johansson, S. (1996). Attendance at cultural events, reading books or periodicals, and making music or singing in a choir as determinants for survival: Swedish interview survey of living conditions. British Medical Journal, vol.313, n.7072, 1577-1580.

Brodel, C. (2003). Early music performance practice for amateur choirs. Musik und Kirche, vol.73, n.2, 106-114.

Brünner, M. (2009). Formación de coros escolares. Eufonía, vol.45, 10-18.

Cámara, A. (2003). El canto colectivo en la escuela: una vía para la socialización y el bienestar personal. Revista de Psicodidáctica, vol.15-16, 105-110.

Carbonell i Guberna, J. (2003). Aportaciones al estudio de la sociabilidad coral en la España contemporánea. Hispania: Revista española de historia vol.63, n.214, 485-504.

Carlow, R. (2006). Diva Irina: An English language learner in high school choir. Bulletin of the council for research in music education, vol.170, 63-77.

Carminatti, J. da S. y Krug, J.S. (2010). A prática de canto coral e o desenvolvimento de habilidades sociais. Pensamiento Psicológico, vol.7, n.14, 81-96.

Clift, S. y Hancox, G. (2001). The perceived benefits of singing: findings from preliminary surveys of a university college choral society. Journal of the royal society for the promotion of health, vol.121, n.4, 248-256.

Daniels, R. (1986). Relationships among selected factors and the sight-reading ability of high-school mixed choirs. Journal of research in music education, vol.34, n.4, 279-289.

De Souza, F. y Santos, M. (2009). O Corpo na música, a música no corpo: A comunidade em um processo de integração. Extensio: Revista Eletrônica de Extensão, vol.6, n.7, 110-116.

Deniz Silva, M. (2001). Orfeonizar a Naçao: O Canto Coral como instrumento educativo e político nos primeiros anos da Mocidade Portuguesa (1936-1945). Revista portuguesa de musicología, vol.11, 139-174.

Dorsey, S. (2011). Choral Journal: An index to Volumes 19-51. Online en http://goo.gl/TEzM2 Consultado el 20 de diciembre de 2011.

Dueck, J. (2008). Mennonite choral music recordings of the West Coast Mennonite Chamber Choir. Journal of American Folklore, vol.120, n.3, 348-360.

Durrant, C. (2009). Communicating accentuating the aesthetic and expressive dimension in choral conducting. International journal of music education, vol.27, n.4, 326-340.

Dwiggins, R. (1984). One-step-at-a-time for show choirs + choreography for high-school-students. Music educators journal, vol.70, n.6, 41-45.

Elorriaga, A. (2011). The construction of male gender identity through choir singing at a Spanish secondary school. International journal of music education, vol.29, n.4, 318-332.

Elsgtröm, E. (2009). La educación vocal en la formación inicial de los docentes al Estado español: descripción de la situación y propuestas de cambio. Temps d'Educació, vol.36, 247-260.

Eyre, L. (2011). Therapeutic Chorale for Persons with Chronic Mental Illness: A Descriptive Survey of Participant Experiences. Journal of Music Therapy, vol.48, n.2, 149-168.

Ferrer Miquel (2009). El canto coral y las orquestas infantiles, una educación en valores. Eufonía, vol. $45,30-38$.

Fiala, J. (1996). Music of the choir of the prestice church in bohemia from the 18th-century to the present. Hudebni Veda, vol.33, n.1, 27-39.

Gackle, L. y Fung, C. (2009). Bringing the East to the West: A Case Study in Teaching Chinese Choral Music to a Youth Choir in the United States. Bulletin of the council for Research in Music Education, vol.182, 65-77.

Gant, A. (2001). In choirs and places where they sing - A survey of contemporary British choral music. Musik und Kirche, vol.71, n.3, 158-164.

Giráldez, H. A. (2007). La Competencia cultural y artística. Madrid: Alianza.

Gómez, A. y Rodilla, F. (2009). Música vocal contemporánea en Extremadura. Junta de Extremadura: Consejería de cultura. 
Gumm, A. (2004). The effect of choral student learning style and motivation for music on perception of music teaching style. Bulletin of the council for research in music education, vol.159, 11-22.

Grant, A., Hutchison, K., Hornsby, D. y Brooke, S. (2008). Creative pedagogies: "Art-full" reading and writing. English teaching-practice and critique, vol.7, n.1, 57-72.

Griffiths, T. (2008). Tone deafness: a model complex cortical phenotype. Clinical Medicine, vol.8, n.6, 592-595.

Gronostay, U. (1980). Music performance, a discordant concept - tasks and goals of a choir director. Neue Zeitschrift fur Musik, vol.141, n.6, 530-532.

Hatem, T., Lira, P. y Mattos, S. (2006). The therapeutic effects of music in children following cardiac suggery. Journal de Pediatria, vol.82, n.3, 186-192.

Heimbucher, C. (2009). The spiritual choir music of Louis Spohr. Musik und Kirche, vol.79, 28-38.

Heald, P. (1996). Reviving the rhetoric of the public interest: Choir directors, copy machines, and new arrangements of public domain music. Duke Law Journal, vol.46, n.2, 241-290.

Hampshire, KR.; Matthijsse, M. (2010). Can arts projects improve young people's wellbeing? A social capital approach. Social Science y Medicine, vol.71, n.4, 708-716.

Hurtado LLopis, J. (2011a). Hacer música para el desarrollo personal y social. Eufonía, vol.51, 24-33. (2006). El desarrollo de la asertividad en la Coral Allegro ONCE Valencia. Eufonía, vol.37, 63-71.

Jaraba, M. (1989). Teoría y práctica del canto coral. Madrid: Istmo.

Jurado Luque, J. (2010). A lenda de Montelongo: A zarzuela galega como manifestación cultural multidisciplinar. Tesis doctoral. La Coruña: Universidad de Coruña.

Kotter, G. (2000). Experiencing music in the flesh. Some trailblazing ideas on a different way of working with choirs. Musik und Kirche, vol.70, n.4, 226-229.

Lobos, A. (2009). In Harmony with Nature - Elements of Ecology in Pedagogy and Art. New Educational Review, vol.19, n.3-4, 383-389.

(2008). 20 Polish Christmas carols for soprano, female choir and orchestra by Witold Lutoslawski as an extremely good example of pedagogical interactions in Polish school of music. New Educational Review, vol.15, n.2, 187-193.

MacLellan, C.R. (2011). Differences in Myers-Briggs Personality Types Among High School Band, Orchestra, and Choir Members. Journal of research in music education, vol.59, n.1, 85-100.

Major, J. (1982). The effect of subdivision activity on rhythmic performance skills in high-school mixed choirs. Journal of research in music education, vol.30, n.1, 31-47.

Marin, R. (1976). Valores, objetivos y actitudes en educación. Valladolid: Miñón.

Morales Villar, Ma . C. (2007). Ramón Guitart-Besangèe y "El arte de desarrollar la voz y sus timbres" (ca. 1915): la escuela de canto de Manuel García en Buenos Aires. En M. Gembero y E. Ros (Eds.), La música y el Atlántico: relaciones musicales entre España y Latinoamérica. Granada: Universidad de Granada.

Omishakin, A., Carlat, J., Hornsby, S. y Buck, T. (2009). Achieving Built-Environment and Active Living Goals Through Music City Moves. American journal of preventive medicine, vol.37, n.6, S412-S419.

Ortega Castejón, J.F. (2005). Formación coral. Murcia: DM.

Ortiz Molina, M ${ }^{a}$ A. (2011a). Tendiendo puentes hacia la interculturalidad... desde Andalucía (España) (I). DEDICA. Revista de Educacao e Humanidades, vol.1, 69-94.

. (2011b). Tendiendo puentes hacia la interculturalidad... desde Andalucía (España) (II). DEDICA. Revista de Educacao e Humanidades, vol.1, 95-111.

Pérez Aldeguer, S. y Leganés, E.N. (2012). La Música como herramienta interdisciplinar: un análisis cuantitativo en el aula de Lengua Extranjera de Primaria. Revista de Investigación en Educación, n.10, vol.1, 127-143.

Phillips, R. (1982). Some perceptions of gospel music. Black perspective in music, vol.10, n.2, 167-178. 
Quijada Sagredo, M. (1985). El canto coral en la tragedia tardía de Eurípides: tópicos de la crítica y nuevas perspectivas. Symbolae Ludovico Mitxelena septuagenario oblatae, vol.1, 179-188.

Quilaqueo Rapimán, D. (2006). Valores educativos mapuches para la formación de persona desde el discurso de kimches. Estudios Pedagógicos, vol. 32, n.2, 73-86.

Reames, R. (2001). High school choral directors' description of appropriate literature for beginning high school choirs. Journal of research in music education, vol.49, n.2, 122-135.

Regelski, A. y Gates, J. (2009). Music Education for Changing Times. New York: Springer.

Ruthmann, A., Heines, J., Greher, G., Laidler, P. y Saulters, C. (2010). Teaching Computational Thinking through Musical Live Coding in Scratch. SIGCSE 10: proceedings of the 41st acm technical symposium on computer science education, 351-355.

Sancho García, M. (2007). Orfeonismo y Canto coral en Valencia (1950-1910). Revista de musicología, vol.30, n.1, 103-126.

Seroff, D. (1990). A brief introduction to the zulu choirs. Black music research journal, vol.10, n.1, 54-47.

Sichivitsa, V. (2003). College choir members' motivation to persist in music: Application of the Tinto model. Journal of Research in Music Education, vol.51, n.4, 330-341.

Sotelo, C. (2009). Les trobades de corals a secundària a Catalunya. Guix: Elements d'acció educativa, vol.360, 26-27.

. (2002). Los encuentros de corales de enseñanza secundaria en Cataluña. Eufonía, vol.26, 115-120.

Schweizer, R. (1989). Elementary music-education and improvisation in the children's choir 2. Musik und Kirche, vol.5, n.2, 67-74.

Stanley, A. (1970). Developing Understanding of Twentieth Century Composition in Junior High School General Music. Department of Heelth, Education, and Wolfere, Office of Education. NewYork: Columbia University.

Teichmann, W. (2003). Gospel music for choirs - An annotated list of source materials. Musik und Kirche, vol.73, n.1, 26.

Terry, K. (1989). Body Music. Musical copyist: Thomas Lawrence McKinley, 10-14. Consultado el 03/01/2012. Disponible en http://goo.gl/0eane

Touriñán, J.M. (2007). Valores y convivencia ciudadana: una responsabilidad de formación compartida y derivada. Bordón, vol.59, n.2-3, 261-311.

Touriñán, J. y Longueira, S. (2010). La música como ámbito de educación. Educación por la música y para la música. Teoría de la educación. Revista interuniversitaria, vol.22, n.2, 151-181.

Walter, T. (1992). Angelic choirs and individual beliefs and personal motives of choir singers, a sociological interpretation of the nonsecularization of choral music. Musical times, vol.133, n.1792, 278-281.

Wills, R. (2011). The magic of music: a study into the promotion of children's well-being through singing. International Journal of Childrens Spirituality, vol.16, n.1, 37-46. 